\title{
Расчетное исследование параметров механической безопасности высотного (404 метра) жилого комплекса «One Tower» в деловом центре «Москва-Сити»
}
А.М.Белостоцкий, НИЦ СтаДи0, Москва
П.А.Акимов, РААСН, НИЦ СтаДи0, Москва
Д.С.Дмитриев, НИЦ СтаДи0, Москва
А.И.Нагибович, НИЦ СтаДи0, Москва
Н.0.Петрашев, НИЦ «Строительство», Москва
С.0.Петряшев, НИЦ «Строительство», Москва

Рассматриваемый в настоящей статье высотный жилой комплекс «One Tower» высотой 403,9 метра будет расположен в Московском международном деловом центре (ММДЦ) «Москва-Сити» и станет одним из самых высоких зданий в Европе. Архитектурный проект уникального объекта был разработан в архитектурном бюро «Сергей Скуратов Architects», конструктивная часть - 000 «Техстрой» (под руководством М.И. Кельмана). Статья посвящена расчётным исследованиям напряжённо-деформированного состояния, прочности и устойчивости несущих конструкций этого многофункционального жилого комплекса с подземной автостоянкой. Приведено краткое описание природных условий района расположения объекта, его несущих конструкций, нагрузок (расчётных и нормативных, основных и особых) и их сочетаний, представлена постановка задач расчётных исследований. Далее кратко описаны использовавшиеся численные методики, алгоритмы и реализующее программноалгоритмическое обеспечение для расчёта статического и динамического напряжённо-деформированного состояния. Представлены разработанная и верифицированная подробная конечноэлементная модель системы «свайное основание - несущие конструкции жилого комплекса», адекватно отражающая его геометрико-жесткостные, инерционные и нагрузочные характеристики и результирующее напряжённо-деформированное состояние, а также результирующие параметры напряжённо-деформированного состояния, прочности и динамики несущих конструкций объекта при нормативно регламентированных сочетаниях вертикальных и ветровых нагрузок, а также расчёты на устойчивость против прогрессирующего обрушения. В заключении на базе выполненных исследований делается обоснованный вывод о достоверности полученных расчётами критериальных параметров, определяющих прочность, устойчивость и динамику несущих конструкций объекта. Все основные расчёты были проведены с использованием лицензионного конечноэлементного программного комплекса ANSYS Mechanical, верифицированного в Российской академии архитектуры и строительных наук (РАACH). Армирование конструкций по конвертированным результирующим усилиям (из расчётов по ANSYS Mechanical) проводилось в программном комплексе «ОМ СНиП Железобетон».
Ключевые слова: математическое моделирование, численное моделирование, компьютерное моделирование, численные методы, метод конечных элементов, механическая безопасность, напряжённо-деформированное состояние, динамические характеристики, высотное здание, «0ne Tower».

Analysis of Parameters of Mechanical Safety of High-Rise (404-meter) Residential Building "One Tower" in the Moscow International Business Center

A.M.Belostotsky, SRC StaDy0, Moscow

P.A.Akimov, RAACS, SRC StaDy0, Moscow

D.S.Dmitriev, SRC StaDy0, Moscow

A.I.Nagibovich, SRC StaDy0, Moscow

N.0.Petryashev, Research Center of Construction, Moscow

S.0.Petryashev, Research Center of Construction, Moscow

It is assumed that the socalled "One Tower" skyscraper with a height of 403.9 meters, considered in the distinctive paper, will be located in the Moscow International Business Center (MIBC) and will become one of the tallest buildings in Europe. The project of a unique object was developed in the architectural bureau "Sergey Skuratov Architects" and "GKTtechStroy" LLC (under the direction of M.I. Kelman). Generally the paper is devoted to computational studies of the stress-strain state, strength and stability ofload-bearing structures of this multifunctional residential complex with an underground parking lot. The paper provides a brief description of the natural conditions of the area of the location of the object, its load-bearing structures, characteristic and design loads, their combinations. Besides, formulation of objectives of computational research is presented. Then numerical methods, algorithms and software, which were used for computational static and dynamic analysis of skyscraper and analysis of corresponding stress-strain state are described. Further, the developed and verified advanced detailed finite element model of the coupled system "pile foundation - loadbearing structures of residential building" is considered. This model adequately reflects corresponding geometric, stiffness, inertial and load parameters and the resulting stress-strain state. Resulting parameters of the stress-strain state, strength and dynamics of load-bearing structures of "One Tower" skyscraper with allowance for design (in accordance with design codes) combinations of vertical and wind loads and progressive 
collapse analysis are under consideration as well. As a result a reasonable conclusion is made about the reliability of the criterion parameters obtained by structural analysis including strength analysis, stability analysis and dynamic analysis of the loadbearing structures of the building. Computational structural analysis was performed with the use of licensed certified finite element software package ANSYS Mechanical, verified by the Russian Academy of Architecture and Construction Sciences (RAACS). Reinforcement of structures was designed in "OM SNiP Zhelezobeton" software after converting of resultant efforts from ANSYS Mechanical.

Keywords: mathematical modelling, numerical modelling, computer modelling, numerical methods, finite element method, mechanical safety, stress-strain state, "0ne Tower" skyscraper.

\section{Постановка задач и исходная информация}

Климат района работ умеренно-континентальный и характеризуется основными показателями, указанными в СП 131.13330.2012 ${ }^{1}$. Инженерно-геологические условия изучаемой площадки относятся к III категории сложности согласно СП 11-105-97², геотехническая категория объекта - III (согласно МГСН 2.07-0133). Геологическая толща, исследованная на глубине до 80,0 метров, состоит из отложений современного отдела четвертичной системы, а также верхне- и среднекаменноугольных отложений.

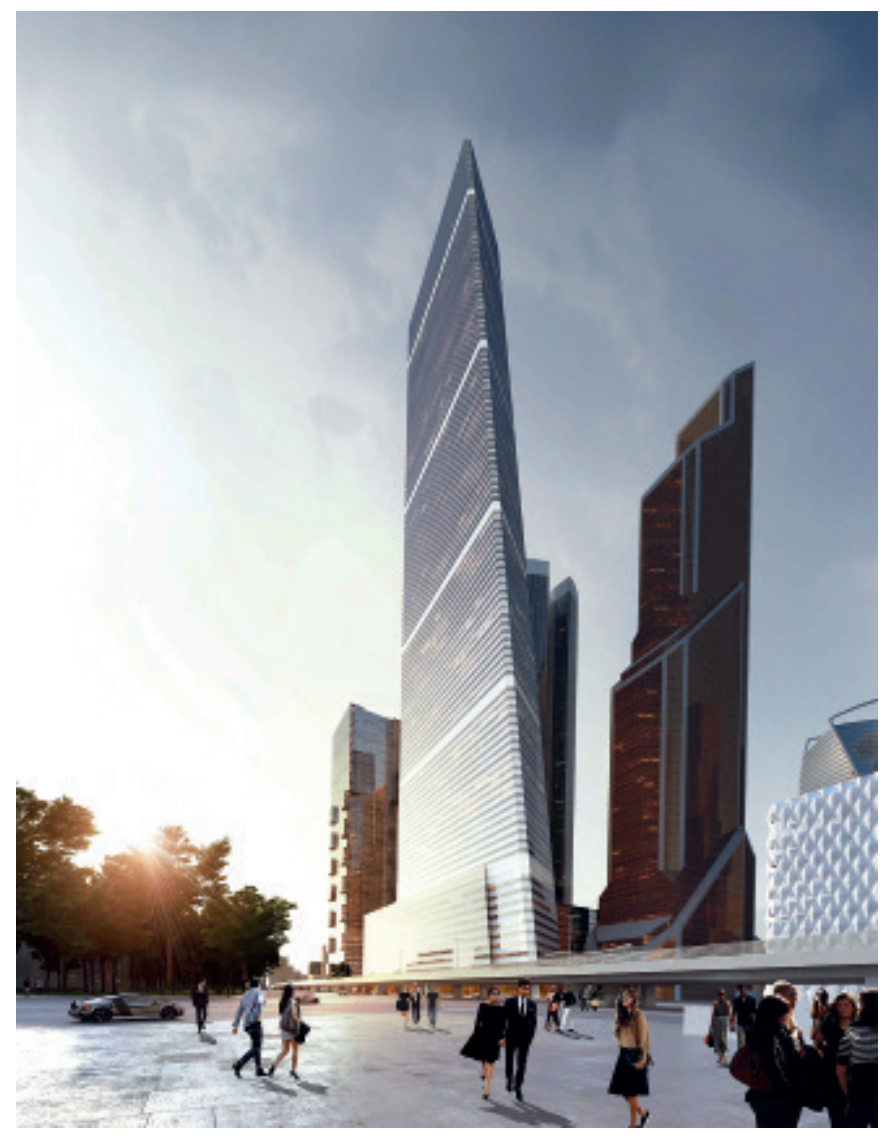

Puc. 1. Внешний вид небоскрёба «One Tower» (рендеринг)
В конструктивном отношении проектируемый объект представляет собой сложный в плане многофункциональный высотный жилой комплекс, состоящий из подземной части (размер в осях 186,9×37,2 м) с четырьмя подземными этажами, стилобатной части (размер в осях 166,95×30,7 м) с количеством этажей - 12, и высотной части (высота 403,9 м над абсолютной отметкой 129,30, размеры в осях 113,4×27 м) с количеством этажей - 92. Конструктивная схема высотной части - монолитный железобетонный каркас с ядром жёсткости и несущими колоннами (рис. 1).

Необходимая степень долговечности конструкций обеспечена комплексом мероприятий, основным из которых является применение качественных материалов (бетона класса В50, 60, 80 арматуры классов A240, A500C), теплоизоляционных материалов, обеспеченных сертификатами, гарантирующими соответствующие сроки эксплуатации.

Пространственная жёсткость и неизменяемость монолитного каркаса здания обеспечиваются совместной работой горизонтальных дисков перекрытий и вертикальных несущих стен ядра, колонн и пилонов каркаса.

Конструктивная схема высотной части - монолитный железобетонный каркас с ядром жёсткости и несущими колоннами. Свайное основание объекта включает 685 свай. Нагрузки, используемые в расчётной модели, были согласованы с заказчиком, сбор нагрузок выполнен в соответствии с СП 20.13330.2011 4 и СП 267.1325800.2016 ${ }^{5}$ и соответствующими специальными техническими условиями (СТУ).

Исходные данные принимались в соответствии с научнотехническим отчётом по теме определения расчётных ветровых нагрузок, действующих на объект (комплексное моделирование обтекания воздушным потоком макета комплекса было проведено с использованием специализированной аэродинамической трубы А-6 НИИ механики Московского государственного университета им. М.В. Ломоносова ${ }^{6}$ и соответствующих нормативных методик).

В соответствии с техническим заданием ${ }^{7}$ были поставлены и решались следующие задачи: анализ и обобщение

${ }^{1}$ СП 131.13330.2012 Строительная климатология. Актуализированная редакция СНиП 23-01-99* (с Изменениями №1, 2). - М.: АО «Кодекс», 2018.

${ }^{2}$ СП 11-105-97 Инженерно-геологические изыскания для строительства. Часть І. Общие правила производства работ. - М.: ПНИИИС Госстроя России, 1997

${ }^{3}$ TCH 50-304-2001 г. Москвы (МГСН 2.07-01) Основания, фундаменты и подземные сооружения. - М., 2003.

${ }^{4}$ СП 20.13330.2011 Нагрузки и воздействия. Актуализированная редакция СНиП 2.01.07-85*. - М. : ФГУП ЦПП, 2011

${ }^{5}$ СП 267.1325800.2016 Здания и комплексы высотные. Правила проектирования. - М. : Стандартинформ, 2017.

${ }^{6}$ Научно-технический отчёт «0пределение расчётных ветровых нагрузок, действующих на проектируемый объект: Многофункциональный жилой комплекс на территории ММДЦ «Москва-Сити» по адресу: г. Москва, Красногвардейский проезд, вл. 13 и вл. 13 стр. 1. Москва. - М. : НИИ механики МГУ им. М.В. Ломоносова, 2018.

${ }^{7}$ Многофункциональный высотный жилой комплекс с подземной автостоянкой на территории ММДЦ «Москва-Сити», участок №1, по адресу: г. Москва, 1-й Красно-гвардейский проезд, вл. 13. Техническое задание на расчётные исследования. - М.: 000 «Архитектурное бюро Сергея Скуратова», 2018. 
проектной документации и данных изысканий, постановка задач расчётных исследований; разработка и верификация расчётных пространственных оболочечно-стержневых конечноэлементных моделей жилого комплекса; определение параметров напряжённо-деформированного состояния (НДС) (перемещения, усилия) и расчётного армирования несущих железобетонных конструкций при нормативно регламентированных сочетаниях вертикальных и ветровых нагрузок; проведение расчётов на устойчивость против прогрессирующего обрушения.

\section{Методика и программное обеспечение расчётов}

Дискретизация по пространству и решение соответствующих задач математического (численного) и компьютерного моделирования состояния рассматриваемого объекта осуществлялись в рамках метода конечных элементов (МКЭ). Соответствующее уравнение движения геометрически линейной системы в матричной форме метода перемещений (в условиях малости перемещений и деформаций) имеет вид $[1 ; 2 ; 3 ; 4 ; 5 ; 6 ; 7 ; 8]$ :

$$
M \ddot{\bar{u}}(t)+C \dot{\bar{u}}(t)+\left(K+K_{G}\right) \bar{u}(t)=\bar{F}(t)+\bar{R}(\bar{u}, \dot{\bar{u}}),
$$

где $M, C, K, K_{\mathrm{G}}$ - соответственно симметрические, положительно определённые, блочноредкозаполненные матрицы масс, демпфирования, жёсткости и геометрической жёсткости конечноэлементной модели (КЭМ) системы; $t$ - время; «точка» означает дифференцирование по времени; $\bar{F}(t)$ - вектор заданных статических и динамических нагрузок; $\bar{R}(\bar{u}, \dot{u})$ - вектор псевдонагрузок, моделирующий физически нелинейные эффекты; $\bar{u}(t)$ - искомый вектор обобщённых динамических перемещений КЭМ.

Отметим, что уравнения (1), учитывающие кинематические граничные условия, доопределяются начальными условиями (из решения статической задачи при $t=t_{0}$ ). Матрицы $K, K_{\mathrm{G}}$, $M, C$ и векторы $\bar{F}$ и $\bar{R}$ строятся на основе соответственно матриц и векторов сосредоточенных факторов, а также матриц и векторов конечных элементов, вычисляемых в общем случае с применением квадратур оптимальной точности. Для адекватной и гибкой аппроксимации геометрико-жесткостных, инерционных и диссипативных свойств, статических и динамических нагрузок и результирующего НДС разнообразных пространственных комбинированных систем разработан представительный набор стержневых, мембранных, плитно-оболочечных, двумерных и трёхмерных (объёмных) конечных элементов, совместимых в единой расчётной модели и допускающих альтернативные процедуры сборки [9; 1].

Общая динамическая постановка (1) естественным образом сводится к важным частным задачам - к статической, на собственные значения (собственные частоты и формы колебаний, критические числа и формы потери начальной устойчивости) и к спектральным формулировкам динамических задач. Для этого используются соответствующие виды нагрузок и решений, выполняется обнуление незначимых матриц.
Для решения системы линейных алгебраических уравнений (СЛАУ) статического равновесия с l вариантами нагрузок:

$$
K\left[\begin{array}{lll}
\bar{u}_{1} & \ldots & \bar{u}_{1}
\end{array}\right]=\left[\begin{array}{lll}
\bar{F}_{1} & \ldots & \bar{F}_{1}
\end{array}\right]
$$

применяется эффективный вариант прямого метода Гаусса для положительно определённых симметричных блочноредкозаполненных матриц (SPARSE-схема), модифицированная схема квадратного корня (Холецкого) или итерационная схема сопряжённых градиентов с предобуславливанием (PCG) [10].

Заметим, что к последовательности задач типа (2) сводятся также нелинейные, нестационарные и (или) динамические задачи $[5 ; 11]$.

Как известно, в рамках спектральных динамических расчётов (в частности, и для анализа пульсационной составляющей ветровой нагрузки) или самостоятельно решается частная проблема собственных значений [5]:

$K \Phi=\Omega^{2} M \Phi$, где $\Phi=\left[\bar{\varphi}_{1} \ldots \bar{\varphi}_{\text {NITER }}\right], \Omega^{2}=\operatorname{diag}\left(\omega_{1}^{2}, \ldots, \omega_{\text {NITER }}^{2}\right)$.

В качестве «базовых» наиболее эффективных подходов к решению обобщённой проблемы собственных значений были выбраны и оптимизированы метод итераций подпространства и блочный метод Ланцоша [8]. Многочисленные вычислительные эксперименты, проведённые авторами, в том числе для плохо обусловленных систем и систем с кратными частотами, позволяют судить о надёжности и эффективности использовавшихся реализаций методов. Заметим, что для больших задач блочный метод Ланцоша имеет существенные преимущества в скорости определения заданного количества собственных частот и форм [10].

Для определения перемещений и усилий в строительных конструкциях от действия постоянной и временной, снеговой и ветровой нагрузок строились и анализировались квазистатические расчётные конечноэлементные модели систем «оболочечно-стержневые конструкции - неоднородное винклеровское основание». Коэффициенты постели упругого основания Винклера принимались по статическим характеристикам грунта.

Динамическая (пульсационная) составляющая ветрового нагружения определялась на основе статической составляющей с учётом коэффициентов динамичности и пульсации, определённых по СП 20.13330.2016².

С сожалением приходится констатировать, что в настоящее время отсутствует обоснованная, внутренне непротиворечивая методика определения критериального параметра - максимального ускорения перекрытий верхних этажей $a_{\max }$ при действии пульсационной составляющей ветровой нагрузки (не должны превышать 0,08 м/ $\mathrm{c}^{2}$ ). Практикуются различные приближённые подходы, сводящиеся, как правило, к использованию формулы:

$$
a_{\max }=u_{\text {max }} \omega^{2},
$$

где $u_{\max }$ - максимальное горизонтальное перемещение; $\omega$ превалирующая собственная частота (рад/с).

${ }^{8}$ СП 20.13330.2016 Нагрузки и воздействия. Актуализированная редакция СНиП 2.01.07-85*. - М. : Стандартинформ, 2018. 
Определение НДС, динамических характеристик и устойчивости несущих конструкций здания выполнялось по верифицированному в Российской академии архитектуры и строительных наук (РАACH) лицензионному программному комплексу (ПК) ANSYS 15.0, реализующему развитые схемы методов конечных элементов и суперэлементов для статических и динамических расчётов пространственных комбинированных систем и поддерживающему все необходимые типы расчётного анализа. Силами ЗАО НИЦ СтаДи0 и ЗАО «НПКТБ ОПТИМИЗАЦИЯ» была выполнена программная разработка, обеспечивающая экспорт значимых геометрических и физико-механических характеристик, «классы» нагрузок и результирующих расчётных усилий основных элементов (колонн, балок, плит, оболочек) железобетонных конструкций из ПК ANSYS в «OM СНиП Железобетон» [12]. В свою очередь последняя программа реализует все расчёты железобетонных конструкций, предусмотренные СНиП 2.03.01-84*9, СНиП 2.05.03-84*10, СП 52-101-2003 ${ }^{11}$, СП 52-102-2004 ${ }^{12}$, СП 52-103-2007 ${ }^{13}$, а также положения проекта актуализированной редакции 2008 года СНиП 2.05.03-84*14. Таким образом была обеспечена и автоматизирована оценку прочности несущих железобетонных конструкций исследуемого здания [12].

\section{Построение и анализ расчётных конечноэлементных моделей здания}

Заказчиком были переданы соответствующие файлы, подготовленные в пакете Autodesk AutoCAD, комплект актуальных

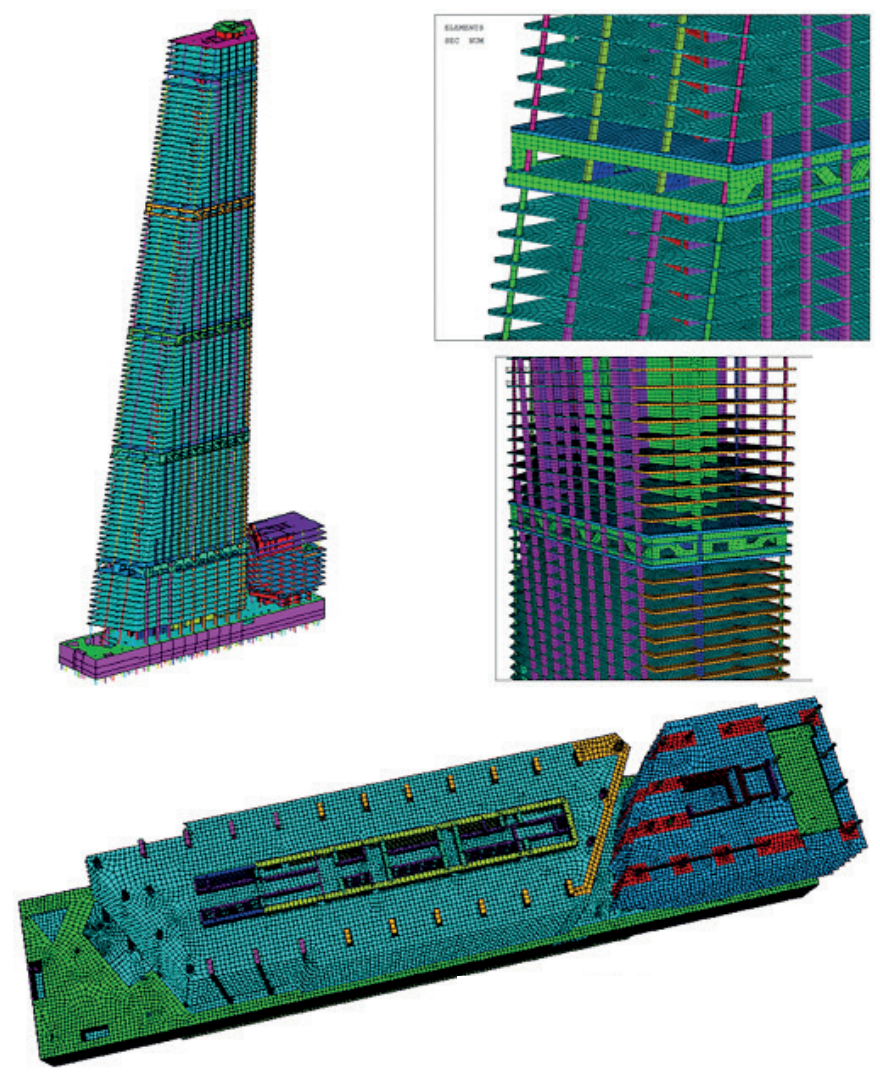

Рис. 2. Конечноэлементная модель объекта и её отдельные фрагменты. Изометрия чертежей и схем многофункционального высотного жилого комплекса. Кроме того, в ЗАО «НИЦ СтаДи0» была передана трёхмерная модель здания в формате Revit. На основании анализа и обобщения этих материалов в программном комплексе ANSYS были построены и верифицированы пространственные оболочечно-стержневые конечноэлементные модели несущих конструкций объекта.

Железобетонные плиты фундамента, перекрытий, несущие стены моделировались плоскими треугольными и четырёхугольными в плане оболочечными конечными элементами (КЭ) постоянной толщины, реализующими гипотезы Кирхгофа-Лява. Железобетонные колонны и балки были представлены пространственными стержневыми КЭ.

КЭ жёстких вставок типа МРС184 с опцией «жёсткая балка» введены в местах стыковки плит перекрытий и фундаментной плиты с колоннами.

В целях задания коэффициента жёсткости основания (Винклера) элементы поверхностных эффектов типа SURF154 были «наклеены» на фундаментную плиту.

Были приняты следующие продольные жёсткости (вертикальные) свай: для статических задач - в соответствии с имевшимися данными Научно-исследовательского, проектно-изыскательского и конструкторско-технологического института оснований и подземных сооружений им. Н.М. Герсеванова АО «Научно-исследовательский центр «Строительство»»; для динамических задач - восьмикратно увеличенные статические жёсткости.

Следует подчеркнуть, что разработанная расчётная модель [13] корректно и адекватно отражала геометрико-жесткостные и инерционные свойства и нагрузочные характеристики строительных конструкций и основания здания. Об этом, в частности, свидетельствовала и вычислительная размерность построенной ANSYS-модели - 613739 узла (3 682434 степеней свободы) и 665350 конечных элементов (рис. 2).

Соответствие геометрико-жесткостных, инерционных и нагрузочных параметров расчётных моделей проектным данным проводилось следующими способами: визуализация и анализ характерных фрагментов и всей модели в различных ракурсах; выдача объёмов, масс и нагрузок характерных фрагментов расчётной модели, их сопоставление с проектными данными и расчётными моделями в альтернативных программных комплексах; анализ результатов статического и динамического расчёта, в частности для предельных случаев, имеющих прозрачный физический смысл [14; 15]. 0тметим, что наиболее информативными верификационными

${ }_{9}^{9}$ СНиП 2.03.01-84* Бетонные и железобетонные конструкции (с Изменениями №1, 2). - М. : ГП ЦПП, 1995

${ }^{10}$ СНиП 2.05.03-84* Мосты и трубы. - М., 2009.

${ }^{11}$ СП 52-101-2003 Бетонные и железобетонные конструкции без предварительного напряжения арматуры. - М. : ГУП «НИИЖБ», ФГУП ЦПП, 2004.

${ }^{12}$ СП 52-102-2004 Предварительно напряжённые железобетонные конструкции. - М. : ФГУП цПП, 2005.

${ }^{13}$ СП 52-103-2007 Железобетонные монолитные конструкции зданий. - М. : ФГУП «НИЦ «Строительство»», 2007.

${ }^{14}$ СНиП 2.05.03-84* Мосты и трубы. - М., 2009 
задачами, интегрирующими многие факторы и параметры расчётной модели и в то же время позволяющими выявить определённые различия, являются соответствующие проблемы определения собственных частот и форм колебаний механических систем.

\section{Результаты расчётных исследований. Заключение}

Анализ результатов расчётных исследований, выполненных по ПК ANSYS Mechanical, показал непротиворечивую картину распределения перемещений и усилий (сил и моментов)
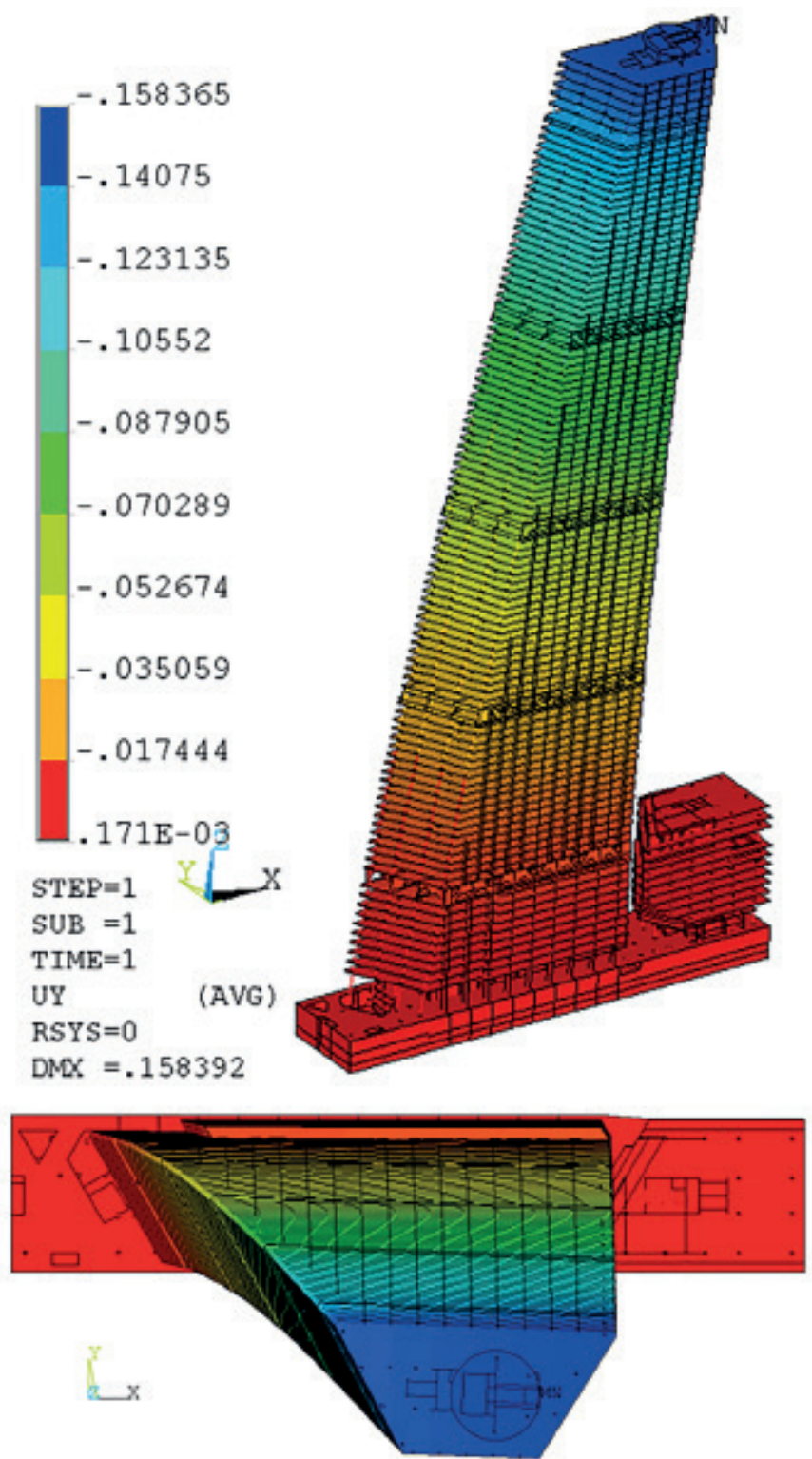

Puс. 3. Горизонтальные амплитуды перемещения $U_{\text {у }}$ (в миллиметрах) от действия пульсационной составляющей ветра с учётом понижающего коэффициента 0,7 (от нормативной нагрузки) и коэффициента надёжности по ответственности 1,15. Основание абсолютно жёсткое. Модуль упругости бетона для вертикальных конструкций был принят начальный, а для горизонтальных - с понижающим коэффициентом 0,3. $U_{\mathrm{y}}=158 \mathrm{MM}$ в фундаменте и каркасе здания при вертикальных статических и ветровых нагрузках, значимых собственных частот и форм колебаний здания.

Критериальные значения кинематических параметров несущих конструкций объекта [прогибы фундаментной плиты, плит перекрытий, покрытия, горизонтальные перемещения верха здания, ускорения верхних этажей (см., например, в частности рис. 3)] находятся в диапазоне нормативно-регламентированных величин:

- максимальный прогиб фундаментной плиты равен 46,3 мм;

- максимальные горизонтальные перемещения от средней составляющей ветровой нагрузки составляют 394 мм;

- максимальное расчётное ускорение перекрытий верхних этажей равно 0,035 м/ $\mathrm{c}^{2}$.

Максимальные усилия в сваях (51011 кН) от расчётного сочетания нагрузок не превышают несущую способность свай $(51200 \mathrm{kH})$.

Выполненными расчётными исследованиями для трёх сценариев инициирующего локального разрушения (рис. 4) наиболее нагруженных несущих конструкций установ-

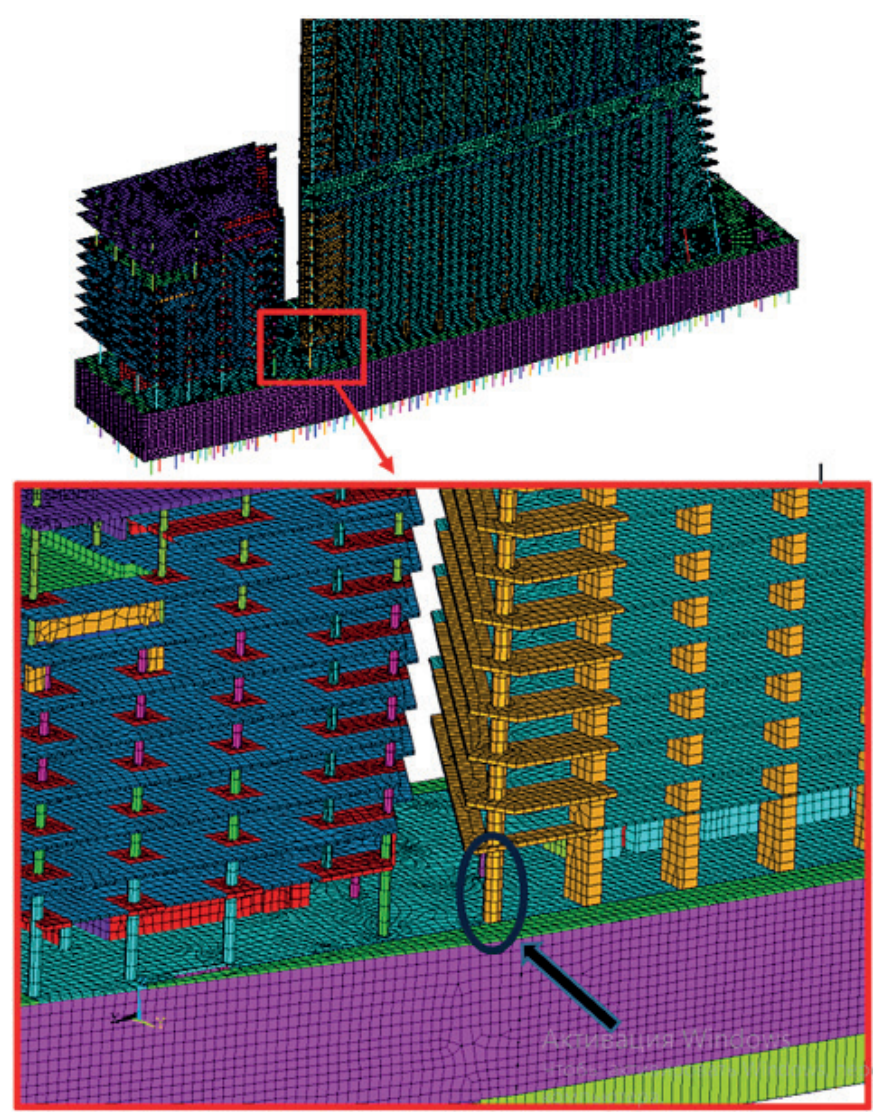

Рис. 4. Фрагмент конечноэлементной модели конструкций. Отмечена разрушенная колонна в одном из сценариев локального разрушения 
лено, что при соблюдении принятых параметров проекта (геометрия, свойства материалов и соединений, величины и сочетания нагрузок и воздействий) и уровне армирования, соответствующего полученным усилиям, железобетонные конструкции каркаса здания устойчивы к прогрессирующему обрушению $[9 ; 16 ; 17 ; 18]$.

Полученные результаты математического моделирования для объекта (статические и ветровые перемещения, собственные частоты и формы колебаний) могут и должны быть также использованы при разработке программы и методики проведения мониторинга состояния основания и несущих конструкций здания в процессе его строительства и эксплуатации $[1 ; 4]$.

\section{Лuтература}

1. Белостоцкий, А.М. Научно-исследовательский центр СтаДи0. 25 лет на фронте численного моделирования / А.М. Белостоцкий, П.А. Акимов // International Journal for Computational Civil and Structural Engineering (Международный журнал по расчёту гражданских и строительных конструкций). - 2016. - Vol. 12, Is. 1. - P. 8-45.

2. Расчётное обоснование механической безопасности стадионов к Чемпионату мира по футболу 2018 года / А.М. Белостоцкий, П.А. Акимов, А.А. Аул [и др.] // Academia. Архитектура и строительство. - 2018. - № 3. - С. 118-129.

3. Numerical simulation of loads and impacts, stress-strain state, strength and stability of unique structures, buildings and facilities. Experience of StaDy0 research \& engineering centre / A.M. Belostotsky, P.A. Akimov, T.B. Kaytukov [et al.] // IOP Conference Series: Materials Science and Engineering. - 2018. - Vol. 456, 012001.

4. Mathematical and Computer Modelling as the Basis of Structural Health Monitoring / T.B. Kaytukov, A.M. Belostosky, P.A. Akimov, V.N. Sidorov // IOP Conference Series: Materials Science and Engineering. - 2018. - Vol. 456, 012072.

5. A review of automatic time-stepping strategies on numerical time integration for structural dynamics analysis / D.F. Rossi, W.G., Ferreira W.J. Mansur, A.F.G. Calenzani // Engineering Structures. - 2014. - Vol. 80. - P. 118-136.

6. Travush, V.I. Contemporary Digital Technologies in Construction. Part 1: About Mathematical (Numerical) Modelling / V.I. Travush, A.M. Belostotsky, P.A. Akimov // IOP Conference Series: Materials Science and Engineering. - 2018. - Vol. 456, 012029.

7. Travush, V.I. Contemporary Digital Technologies in Construction. Part 2: About Experimental \& Field Studies, Material Sciences, Construction Operations, BIM and "Smart" City / V.I. Travush, A.M. Belostotsky, P.A. Akimov // IOP Conference Series: Materials Science and Engineering. - 2018. - Vol. 456, 012030.

8. Accurate and fast three-dimensional free vibration analysis oflarge complex structures using the finite element method / J. Yin, L. Xu, H. Wang [et al.] // Computers \& Structures. - 2019. - Vol. 221. - P. 142-156.
9. Барабаш, М.С. Моделирование жизненного цикла конструкций высотных зданий с учётом сопротивляемости прогрессирующему разрушению / М.С. Барабаш // International Journal for Computational Civil and Structural Engineering (Международный журнал по расчёту гражданских и строительных конструкций). - 2013. - Vol. 9, Is. 4. - Р. 101-106.

10. Расчетные исследования напряженно-деформированного состояния, прочности и устойчивости несущих конструкций высотного здания с учетом фактического положения железобетонных конструкций / А.М. Белостоцкий, П.А. Акимов, Н.О. Петряшев [и др.] // Вестник МГСУ. - 2015. - № 4. - C. 50-68.

11. Wang $L$. A time finite element method for structural dynamics / Wang L., Zhong H. // Applied Mathematical Modelling. - 2017. - Vol. 41. - pp. 445-461.

12. Аул, А.А. Расчёт железобетонных конструкций при совместном использовании программ ANSYS и «OM СНиП Железобетон» / А.А. Аул, А.М. Белостоцкий, М.Б. Краковский // Бетон и железобетон. - 2011. - № 5. - С. 19-23.

13. Ляхович, Л.С. Роль парадоксов в оценке корректности расчетных моделей / Л.С. Ляхович, А.В. Перельмутер, В.И. Сливкер // International Journal for Computational Civil and Structural Engineering / Международный журнал по расчету гражданских и строительных конструкций. - 2013. - Vol. 9, Iss. 2. - P. 34-42.

14. Mechanical Safety and Survivability of Buildings and Building Structures under Different Loading Types and Impacts /V. Travush, S. Emelianov, V. Kolchunov, A. Bulgakov // Procedia Engineering. - 2016. - Vol. 164. - P. 416-424.

15. Travush, V.I. Computer Modeling as Evaluation Method of Column Base Bearing Capacity in Tower Buildings / V.I. Travush, A.S. Martirosyan, G.G. Kashevarova // Procedia Engineering. 2016. - Vol. 153. - P. 773-780.

16. Кашеварова, Г.Г. Исследование проблемы защиты типовых жилых зданий от прогрессируюшего разрушения / Г.Г. Кашеварова, А.А. Пепеляев// International Journal for Computational Civil and Structural Engineering / Международный журнал по расчету гражданских и строительных конструкций. - 2008. - Vol. 4, Iss. 2. - P. 69-70.

17. Progressive collapse analysis and structural robustness of steel-framed modular buildings / S.-C. Lin ,Y. Bai, J. Hou, Y. Huang // Engineering Failure Analysis. - 2019. - Vol. 104. - P. 643-656.

18. Wilkes, J. An energy flow approach for progressive collapse assessment / J. Wilkes, T. Krauthammer // Engineering Structures. - 2019. - Vol. 190. - P. 333-344.

\section{References}

1. Belostotskii A.M., Akimov P.A. Nauchno-issledovatel'skii tsentr StaDi0. 25let na fronte chislennogo modelirovaniya [25th anniversary of Scientific Research Center StaDy0]. International Journal for Computational Civil and Structural Engineering, 2016, Vol. 12, Is. 1, pp. 8-45. (In Russ., abstr. in Engl.) 
2. Belostotsky A.M., Akimov P.A., Aul A.A., Dmitriev D.S., Dyadchenko Yu.N., Nagibovich A.I., Ostrovskii K.I., Pavlov A.S. Raschetnoe obosnovanie mekhanicheskoi bezopasnosti stadionov k Chempionatu mira po futbolu 2018 goda [Analysis of Mechanical Safety of Stadiums for the World Cup 2018]. Academia. Arkhitektura i stroitel'stvo [Academy. Architecture and construction], 2018, no. 3, pp. 118-129. (In Russ., abstr. in Engl.)

3. Belostotsky A.M., Akimov P.A., Kaytukov T.B., Pavlov A.S., Aul A.A., Afanasyeva I.N., Vershinin V.V., Dmitriev D.S., Dyadchenko Yu.N., Nagibovich A.I., Ostrovsky K.I., Petryashev S.0., Petryashev N.0., Scherbina S.V., Chauskin A.S., Goryachevsky 0.S., Negrozov 0.A. Numerical simulation of loads and impacts, stress-strain state, strength and stability of unique structures, buildings and facilities. Experience of StaDy0 research \& engineering centre. IOP Conference Series: Materials Science and Engineering, 2018, Vol. 456, 012001. (In Engl.)

4. Kaytukov T.B., Belostosky A.M., Akimov P.A., Sidorov V.N. Mathematical and Computer Modelling as the Basis of Structural Health Monitoring. IOP Conference Series: Materials Science and Engineering, 2018, Volume 456, 012072. (In Engl.)

5. Rossi D.F., Ferreira W.G., Mansur W.J., Calenzani A.F.G. A review of automatic time-stepping strategies on numerical time integration for structural dynamics analysis // Engineering Structures, 2014, Vol. 80, pp. 118-136. (In Engl.)

6. Travush V.I., Belostotsky A.M., Akimov P.A. Contemporary Digital Technologies in Construction Part 1: About Mathematical (Numerical) Modelling. IOP Conference Series: Materials Science and Engineering, 2018, Vol. 456, 012029. (In Engl.)

7. Travush V.I., Belostotsky A.M., Akimov P.A. Contemporary Digital Technologies in Construction Part 2: About Experimental \& Field Studies, Material Sciences, Construction Operations, BIM and "Smart" City. IOP Conference Series: Materials Science and Engineering, 2018, Vol. 456, 012030. (In Engl.)

8. Yin J., Xu L., Wang H., Xie P., Huang S., Liu H., Yang Z., Li B. Accurate and fast three-dimensional free vibration analysis oflarge complex structures using the finite element method. Computers \& Structures, 2019, Vol. 221, pp. 142-156. (In Engl.)

9. Barabash M.S. Modelirovanie zhiznennogo tsikla konstruktsii vysotnykh zdanii s uchetom soprotivlyaemosti progressiruyushchemu razrusheniyu [Modeling the life cycle high-rise buildings structures in view resistance progressive destruction]. International Journal for Computational Civil and Structural Engineering, 2013, Vol. 9, Is. 4, pp. 101-106. (in Russ.)
10. Belostotskii A.M., Akimov P.A., Petryashev N.0., Petryashev S.0., Negrozov 0.A. Raschetnye issledovaniya napryazhenno-deformirovannogo sostoyaniya, prochnosti i ustoichivosti nesushchikh konstruktsii vysotnogo zdaniya s uchetom fakticheskogo polozheniya zhelezobetonnykh konstruktsii [Strength and stability analysis of load-bearing structures of a high-rise building with account for actual positions of reinforced concrete structural members]. Vestnik MGSU [Vestnik MGSU], 2015, no. 4, pp. 50-68.

11. Wang L., Zhong H. A time finite element method for structural dynamics. Applied Mathematical Modelling, 2017, Vol. 41, pp. 445-461.

12. Aul A.A., Belostotsky A.M., Krakovskii M.B. Raschet zhelezobe-tonnykh konstruktsii pri sovmestnom ispol'zovanii programm ANSYSi «OMSNiP Zhelezobeton» [Analysis of reinforced concrete structures with the joint use of the ANSYS and "OM SNiP Zhelezobeton" software systems]. Beton i zhelezobeton [Concrete and reinforced concrete], 2011, no. 5, pp. 19-23. (in Russ.)

13. Lyakhovich L.S., Perel'muter A.V., Slivker V.I. Rol' paradoksov $v$ otsenke korrektnosti raschetnykh modelei [Role of paradoxes when estimating the correctness of design models]. International Journal for Computational Civil and Structural Engineering, 2013, Vol. 9, Is. 2, pp. 34-42. (in Russ.)

14. Travush V., Emelianov S., Kolchunov V., Bulgakov A. Mechanical Safety and Survivability of Buildings and Building Structures under Different Loading Types and Impacts. Procedia Engineering, 2016, Vol. 164, pp. 416-424.

15. Travush V.I., Martirosyan A.S., Kashevarova G.G. Computer Model-ing as Evaluation Method of Column Base Bearing Capacity in Tower Buildings. Procedia Engineering, 2016, Vol. 153, pp. 773-780.

16. Kashevarova G.G., Pepelyaev A.A. Issledovanie problemy zashchity tipovykh zhilykh zdanii ot progressiruyushego razrusheniya [Comprehensive view on the topic of protection of buildings against progressive collapse]. Inter-national Journal for Computational Civil and Structural Engineering, 2008, Vol. 4, Is. 2, pp. 69-70. (in Russ.)

17. Lin S.-C., Bai Y., Hou J., Huang Y. Progressive collapse analysis and structural robustness of steel-framed modular buildings. Engineering Failure Analysis, 2019, Vol. 104, pp. 643-656.

18. Wilkes J., Krauthammer T. An energy flow approach for progressive collapse assessment. Engineering Structures, 2019, Vol. 190 , pp. 333-344.

Белостоцкий Александр Михайлович (Москва). Доктор технических наук, профессор, член-корреспондент РААСН. Генеральный директор Научно-исследовательского центра «СтаДи0» (125124, Москва, ул. 3-я Ямского Поля, д.18, оф. 810. НИЦ «СтаДи0»). Эл.почта: amb@stadyo.ru.

Акимов Павел Алексеевич (Москва). Доктор технических наук, профессор, академик РААСН. Главный ученый секретарь Российской академии архитектуры и строительных наук (107031, Москва, ул. Большая Дмитровка, 24. РААСН), заместитель директора по науке Научно-исследовательского цен-тра «СтаДи0». Эл.почта: akimov@raasn.ru. 
Дмитриев Дмитрий Сергеевич (Москва). Ведущий инженер-расчетчик отдела расчетных исследований Научно-исследовательского центра «СтаДи0» (125124, Москва, ул. 3-я Ямского Поля, д.18, оф. 810). Эл.почта: stadyo@stadyo.ru.

Нагибович Александр Игоревич (Москва). Ведущий инженер-расчетчик отдела расчетных исследований Научно-исследовательского центра «Ста-Ди0» (125124, Москва, ул. 3-я Ямского Поля, д.18, офис 810). Эл.почта: stadyo@stadyo.ru.

Петряшев Николай Олегович (Москва). Инженер Центрального научно-исследовательского института строительных конструкций (ЦнИИСК) им. В.А. Кучеренко Акционерного общества «Научно-исследовательский центр “Строительство”» (109428, г. Москва, ул. Институтская 2-я, д. 6. ЦниИСК им. В.А. Кучеренко). Эл.почта: petryashev.n.o@gmail.com.

Петряшев Сергей Олегович (Москва). Инженер Центрального научно-исследовательского института строительных конструкций (ЦниИСК) им. В.А. Кучеренко Акционерного общества «Научно-исследовательский центр “Строительство”» (109428, г. Москва, ул. Институтская 2-я, д. 6. ЦниИСК им. В.А. Кучеренко). Эл.почта: petsero@mail.ru.

Belostotsky Alexander Mikhailovich (Moscow). Doctor of Technical Sciences, Professor, Corresponding Member of RAACS. General Director of the Research Center "StaDi0" (18 3-ya Yamskogo Polya St, office 810, Moscow, 125124. SRC StaDy0). E-mail: amb@ stadyo.ru.

Akimov Pavel Alekseevich (Moscow). Doctor of Technical Sciences, Professor, Academician of the RAACS. Chief Scientific Secretary at the Russian Academy of Architecture and Construction Sciences (24 Bolshaya Dmitrovka St, Moscow, 107031, RAACS), Deputy Director for Science of the Research Center StaDi0 (18 3-ya Yamskogo Polya St, office 810, Moscow, 125124. SRC StaDy0). E-mail: akimov@raasn.ru.

Dmitriev Dmitry Sergeevich (Moscow). Leading Engineer-Calculationalist at the Department of Computational Research of the Research Center "StaDi0" (18 3-ya Yamskogo Polya St, office 810, Moscow, 125124). E-mail: stadyo@stadyo.ru.

Nagibovich Alexander Igorevich (Moscow). Leading Engineer-Calculationalist at the Department of Computational Research of the Research Center "StaDi0" (18 3-ya Yamskogo Polya St, office 810, Moscow, 125124). E-mail: stadyo@stadyo.ru.

Petryashev Nikolay Olegovich (Moscow). Engineer of the Research Institute of Building Constructions (TSNIISK) named after V.A. Koucherenko A0 "Scientific-Research Center "Construction" (6 2-ya Institutskaya St, Moscow, 109428. TSNIISK). Email: petryashev.n.o@gmail.com.

Petryashev Sergey Olegovich (Moscow). Engineer of the Research Institute of Building Constructions (TSNIISK) named after V.A. Koucherenko A0 "Scientific-Research Center "Construction" (6 2-ya Institutskaya St, Moscow, 109428. TSNIISK). E-mail: petsero@mail.ru. 